\title{
Efficacy of Combining Hyaluronic Acid and Platelet-Rich Fibrin in Diabetic Foot Ulcer
}

\author{
Ronald W. Kartika ${ }^{1}$, Idrus Alwi ${ }^{2}$, Em Yunir ${ }^{2}$, Sarwono Waspadji ${ }^{2}$, Franciscus D. \\ Suyatna $^{3}$,Saptawati Bardosono ${ }^{4}$, Suzzana Immanuel ${ }^{5}$, Saleha Sungkar ${ }^{6}$, Jusuf \\ Rachmat $^{7}$, Todung Silalahi ${ }^{8}$, Mirta Hediyati Reksodiputro ${ }^{9, *}$ \\ ${ }^{1}$ Doctoral Program in Medical Science Faculty of Medicine Universitas Indonesia, Department of Thoracic, Cardiac and Vascular \\ Surgery , Faculty of Medicine Krida Wacana Christian University; ${ }^{2}$ Department of Internal Medicine, Faculty of Medicine Universitas \\ Indonesia - Cipto Mangunkusumo Hospital, Jakarta, Indonesia ; ${ }^{3}$ Department of Clinical Pharmacology, Faculty of Medicine Universitas \\ Indonesia; ${ }^{4}$ Department of Clinical Nutrition , Faculty of Medicine Universitas Indonesia, Jakarta, Indonesia ${ }^{5}$ Department of Clinical \\ Pathology, Faculty of Medicine Universitas Indonesia - Cipto Mangunkusumo Hospital, Jakarta, Indonesia; ${ }^{6}$ Department of Parasitology , \\ Faculty of Medicine Universitas Indonesia, Jakarta, Indonesia; ${ }^{7}$ Department of Thoracic Cardiac and Vascular Surgery , Faculty of \\ Medicine Universitas Indonesia, Jakarta, Indonesia; ${ }^{8}$ Department of Internal Medicine, Faculty of Medicine Krida Wacana Christian \\ University , Jakarta, Indonesia; ${ }^{9}$ Department of Otorhinolaryngology, Facial Plastic Reconstructive Division, Faculty of Medicine, \\ Universitas Indonesia, Cipto Mangunkusumo Hospital, Jakarta, Indonesia.
}

Received: March 3, 2021; Revised: May 2, 2021; Accepted: July 19, 2021

\begin{abstract}
Objectives: A chronic complication of type-2 diabetes mellitus (DMT-2) is Diabetic Foot Ulcer (DFU). The main treatment used in DFU is wound cleansing, followed by dressing the wound as a local control to increase tissue granulation and epithelialization. This study aims to compare the efficacy of the combination of Hyaluronic Acid with Platelet Rich Fibrin (HAPRF) and Platelet Rich Fibrin alone in DFU.

Methods: We conducted a randomized controlled trial from July 2019 to April 2020. The study was approved by the Ethics Committee of the Faculty of Medicine of Universitas Indonesia ID 0855 / UN2.F1 / ETIK / 2018. Informed consent was obtained from the patients.

This was a randomized clinical study to compare the efficacy of HAPRF and PRF in DFU one week post debridement. Twenty DFU samples were collected divided into 2 treatment groups: topical treatment using HAPRF compare with PRF alone. Assessment for wounds improvement was recorded using a digital camera 48 mega pixel with an accuracy of $0.1 \%$ on day- $0,3,7$, and 14 . The results of the wound photographs were processed using ImageJ software. The granulation area (GA) and wound area (WA) were evaluated by IBM SPSS software v.20. The general data description was presented in median (range) value and parameter's differences were conducted using Mann-Whitney test

Results: The two treatment groups showed insignificant difference in characteristics between both group before intervention. The mean granulation width after two weeks of use HAPRF was $97.4 \%$ and PRF was $81.9 \%$. Statistical analysis using Mann Whitney test showed granulation area of HAPRF group was significantly different compared with PRF group on day3(p=0.047) , day-7 $(\mathrm{p}=0.004)$ and day-14 ( $\mathrm{p}<0.001)$. At the end of the wound healing process, the HAPRF group was significantly different compared with PRF group on $\Delta$ day $0-3(\mathrm{p}=0.048), \Delta$ day $0-7(\mathrm{p}=0.039)$, and $\Delta$ day $0-14(\mathrm{p}=$ 0.023).
\end{abstract}

Conclusions: HAPRF improves wound healing rate through increasing granulation tissue and epithelialization compared with PRF only in diabetic foot ulcer after 2 weeks post debridement compared to PRF.

Keywords: Diabetic ulcer, Hyaluronic Acid, Platelet Rich Fibrin, Granulation Area, Delta Wound Area

\section{Introduction}

Diabetic foot ulcer (DFU) is one of diabetes mellitus type-2 (T2DM) complications causing tissue damage that is difficult to heal. In the last decade, diabetic ulcer became serious problem, in both the medical and economic fields, and estimated around $15 \%$ offering in DMT2. This situation will increase morbidity and mortality (Amstrong D, 2017)
Standardized therapy previously used for diabetic ulcer includes optimal blood glucose levels control, debridement sharply, offloading/pressure reduction, antibiotic infection controlled, ischemic correction, and wound dressing ( Abott CA, 2002).

Chronic wound healing processes such as DFU are different from acute wounds and require more complex management. The moist atmosphere in DFU increases the rate of granulation formation and epithelialization as well wound repair (Jeffcoate WJ et al. 2018) The principle of

\footnotetext{
* Corresponding author e-mail: citamirta@yahoo.com.
} 
treating wounds with moist was introduced in 1960, and since then much research was carried out by applying the gel on chronic wound treatment (Mill et al. 2014). The Hyaluronic Acid (HA) compound will bind to the extracellular matrix tissue which can help the wound healing process, and support tissue regeneration. HA has been used for a long time in ophthalmology connective tissue diseases, joints inflammatory and rheumatoid arthritis (Price RD et al. 2005).

Previous studies on the use of HA for acute wound care showed faster healing process. Meanwhile for chronic wound such as DFU, there was not much evidence to support this (Kartika et al, 2020). Platelet Rich Fibrin (PRF) contained source of growth factor (GF) obtained alpha granule (Reksodiputro, 2018). Another study states that hyaluronic acid (HA) is a biopolymer contained in the extracellular matrix of the bone, cartilage and skin tissue. Accordingly, HA and platelet concentrate are involved in the pathophysiology underlying wound healing. The aim of this study was to evaluate the clinical efficacy by measuring the development of ulcer area as well as looking at the safety by looking epithelialization signs from HA+PRF and PRF alone in the treatment of Diabetic Foot Ulcer. Research on the use of HA+PRF combination to treat diabetic ulcers has not been fully reported (Shi, 2018).

\section{Methods}

This study had been approved by The Institutional Board of the Faculty of Medicine Universitas, Indonesia (No. 0855/UN2.F1/ETIK/2018). This open-label randomized controlled trial was conducted at Koja District Hospital and Gatot Soebroto Hospital from July 2019 to April 2020.

\subsection{Study Subjects}

DFU subjects age $>18$ years old, suffer chronic wound (more than 4 weeks) on lower limbs. Wagner-2 criteria, and ulcer size $<40 \mathrm{~cm}^{2}$ were recruited and randomly assigned for HA+ PRF group, PRF group and control group. Subjects including International Working Group on the Diabetic Foot (IWGDF) score infection $>2$ ( moderate- high infection), platelet level $<15010^{3} / \mu \mathrm{L}$, Hemoglobin A1C (HbA1c) $>12.0 \%$, impaired kidney function hemophilia, sickle cell anemia, leukemia, peripheral arterial disease, or with incomplete data were excluded. On day-0, day-3 and day-7, fluid samples were collected using cotton swabs and photographs were taken. The examination was performed at the Integrated Laboratory, Faculty of Medicine, Universitas Indonesia.

\section{2. $P R F$ and $H A+P R F$ Production}

To generate PRF and HA+PRF, from the blood peripheral collection and proceed to produce platelet rich fibrin (PRF), each patient had around 20 cc cubit vein from each patient's arm without anticoagulant tube and centrifuge 200G around 8 minutes. We separated fibrin and buffy coat by sterile scissor and applied the fibrin to the wound ( Dohan, 2009). In HAPRF group, process continued to make homogenous HA+PRF by adding HA $0.075 \%$ to PRF with comparison 0.6 cc: 1 cc use vortex machine. Every three days, the same protocol was used, and a picture was taken to evaluate wound assessment.

\subsection{Application of PRF or $H A+P R F$ in DFU}

The wound was first cleaned and debrided. Assessment for Wound Area (WA) and Granulation Area (GA) were made before any fibrin gel application, recorded as day-0. After the assessment, $1 \mathrm{~mL}$ of fibrin gel (HA+PRF, or PRF alone) was applied topically on the wound area of 10 $\mathrm{cm}^{2}$. A sterile gauze was then applied to cover the wound as a secondary dressing to maintain moisture. The treatments were applied for 3 times on day-0, 3 and 7 . After day-7, only a standard $\mathrm{NaCl} 0.9 \%$ therapy was given to the subjects until day-14

\subsection{Assessment for Wounds Improvement}

The wound's area improvement was recorded using a digital camera, 48 mega pixels with an accuracy of $0.1 \%$ on day- $0,3,7$, and 14 . The results of the wound photographs were processed using Image-J (National Institutes of Health, Bethesda, MD, USA), and the GI was evaluated. GI was counted as the ratio between granulation area to wound area, in percent.

\subsection{Statistical Analysis}

IBM SPSS software v.20 (IBM Coorporation, Armonk, NY, USA) was used for all statistical analysis. Statistical significance was determined at the $5 \%$ level. The general data description was presented in mean $\pm \mathrm{SD}$ and the median (range) value. The parameter's differences were conducted using Mann-Whitney u test and independent ttest and the graphs created using GraphPath7 software.

\section{Results}

We got around 25 DFU patients, meanwhile only 20 subjects got included in this study in which 8 (40\%) were male and 12 were $(60 \%)$ female. Twenty subjects with DFU were involved in this study. The subjects were randomly divided into two groups according to fibrin gel applied (HAPRF and PRF alone). HAPRF group had five women and five men, while the PRF group had six women and four men. The subjects' characteristics were already presented in our previous publication (Kartika, 2020). There were no significance differences between the two groups' characteristics.

The effect of topical therapy of both groups was evaluated during two weeks after treatment by measuring the width of granulation tissue. Table 1 shows that there was a significant increase in granulation tissue area in HAPRF group compared with PRF alone in day- $3(\mathrm{p}=$ 0.047), day-7 ( $p=0.004)$ and day-14 ( $p<0.001)$ Furthermore, Accordingly, Figure 1 shows the increase of granulation area in HAPRF compared with PRF in day-3, day-7 and day-14.

Table 1. Mean Presentation of Granulation Area Based on Intervention

\begin{tabular}{lccl}
\hline Intervention & HAPRF & \multicolumn{1}{c}{ PRF } & p- value \\
\hline Day-0 & $42.1(18.4-57.8)$ & $34.8(14.1-58.9)$ & 0.921 \\
Day-3 & $62.2(33.6-81.3)$ & $47.7(28.3-73.0)$ & $0.047^{*}$ \\
Day-7 & $78.9 .1(90.8-65.8)$ & $64.6(37.2-69.9)$ & $0.004^{* *}$ \\
Day-14 & $97.7(89.4-99.6)$ & $79.2(46.0 .4-81.9)$ & $<0.001^{* * *}$ \\
\hline
\end{tabular}

*Data median (min-max), Mann Whitney test 


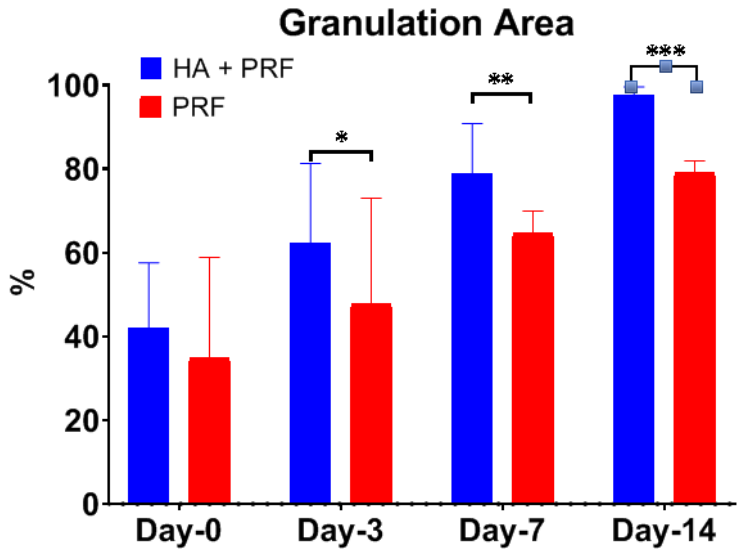

Figure 1. The increasing of granulation area between HAPRF and PRF

Furthermore, this study examined epithelialization growth by measuring wound area in day-3 until day -14 . Table 2 shows that in the HAPRF group there was a significant reduction in wound area compared to PRF alone in day-3 (0.049), day-7 ( $p=0.039)$ dan day-14 $(\mathrm{p}=$ 0.025)

Table 2. Mean of Wound Area ( WA) between the intervention

\begin{tabular}{llll}
\hline Intervention & \multicolumn{1}{c}{$\begin{array}{l}\text { HA+PRF } \\
(\mathrm{n}=10)\end{array}$} & $\begin{array}{l}\text { PRF } \\
(\mathrm{n}=10)\end{array}$ & $\begin{array}{l}\text { p-value } \\
\end{array}$ \\
\hline Before $^{*}$ & $31.9(21.9-39.9)$ & $32.7(29.3-9.7)$ & 0.848 \\
Day-3* $^{* *}$ & $21.7(15.6-31.2)$ & $30.4(21.4-6.5)$ & 0.049 \\
Day-7 $^{* *}$ & $14.9(8.6-16.3)$ & $21.6(24.4-5.6)$ & 0.039 \\
Day-14 $^{* * *}$ & $8.1(6.6-11.6)$ & $15.6(19.0-3.0)$ & 0.025 \\
\hline$\Delta$ day 0-3 & $-10.2(-6.3--8.7)$ & $-2.3(-7.9--3.2)$ & 0.048 \\
$\Delta$ day 0-7 & $-17.9(-13.3--5.9)$ & $-11.1(-4.9--24.1)$ & 0.039 \\
$\Delta$ day 0-14 & $-23.8(-15.3--23.6)$ & $-17.1(-10.3--6.7)$ & 0.023 \\
\hline
\end{tabular}

* Data median (min-maks), Mann Whitney test

There is a decrease of wound area measurement in the wounds that use HA+PRF as topical therapy in DFU (Figure 2). Figure 3 shows the DFU which use topical HA+PRF has wound measurement smaller than the use of topical PRF only.

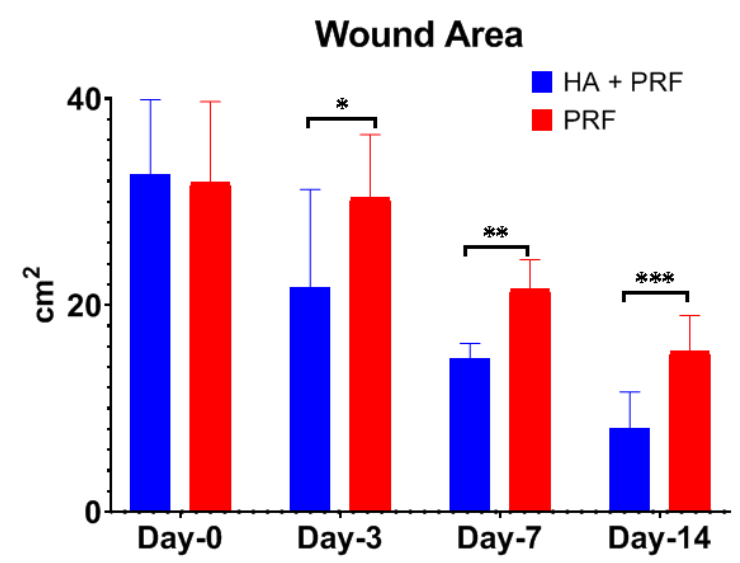

Figure 2. Mean of Wound Area (WA) showed the decrease of wound area between HAPRF vs PRF

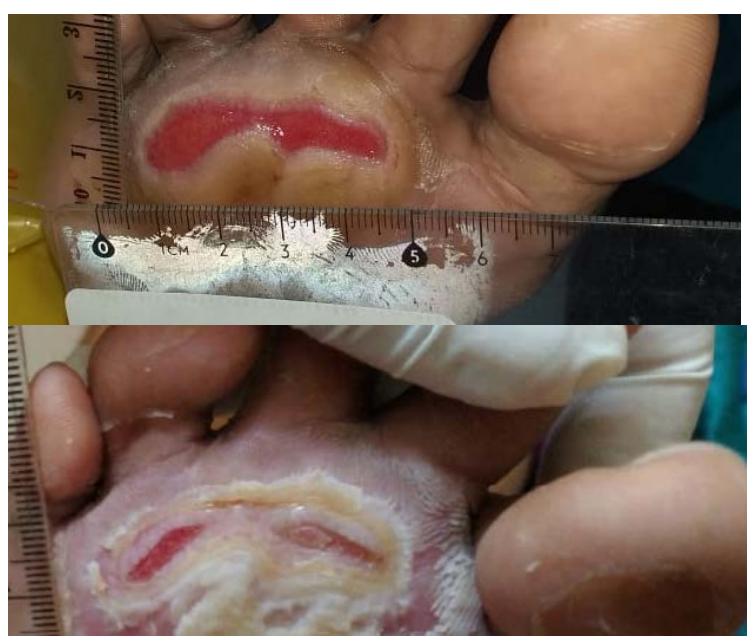

Figure 3. HA+PRF topical in DFU ( Before and after treatment)

\section{Discussion}

In the last decade, diabetic foot ulcers have been a serious problem because chronic wound care is needed with many complications that arise. About 16 million people in the US have T2DM, of which 15\% have complications of DFU, while $12 \%$ of them undergo amputation. According Mill Jr 2014, the process of healing chronic wounds such as diabetic foot ulcers is more complicated so additional therapy may be considered as the therapy of choice. Topical therapy requirements must be able to protect the DFU surface from infection contamination and keep the wound moist so that it can produce faster granulation and epthelialization (Shi , 2018).

In presentation of granulation tissue, it was found that in the HAPRF group, there was an increase of granulation tissue formation in day-7 (83.1\%) and day-14 ( 97.4\%). In the PRF group, there was also an increase in the granulation tissue formation in day-7 (68.3\%) and day-14 (81.9\%). There was a significant difference in the granulation tissue formation in HAPRF group compared with PRF only in day-3 day=7 and day-14 ( $<<0.05$, Mann Whitney test ).

In this study, there were many growth factors trapped in HAPRF compared to PRF alone. Although in PRF alone, there were concentrates of immune and platelet growth factors trapped in the fibrin membrane that induce healing and immunity, but with the addition of HA, there would be an induction effect of growth factor from platelet alpha granules as an anti-inflammatory. It would increase the granulation tissue formation in DFU healing adan induce tissue response growth and tissue regeneration. Many biologically active substances are contained in platelet concentrates and affect tissue repair mechanisms such as cell proliferation, differentiation, and chemotaxis. In addition, there was also an increase in intracellular matrix deposition, angiogenesis substance, immune modulation, antimicrobial activity, and wound tissue remodeling (Sudmann EA 2014).

Platelet-rich fibrin supports three key wound healing mechanisms of "angiogenesis", "immunity", and "epithelial proliferation", and thus implies their use to protect open wounds and promote healing (Pochini, 2016). 
PRF releases any many growth factors namely Transformation Growth Factor $\beta$ - 1 (TGF $\beta-1$ ), Platelet Derivate Growth Factor-AB (PDGF-AB), Vascular Endothelial Growth Factor (VEGF), and other important angiogenesis factor such as matrix cellular glycoproteins Thrombospondin-1 for 7 days ( Dohan, 2009). It is believed that GF is contained in platelets concentration seven times higher compared with non-diabetic patients. In addition, PRF also contains EGF, FGF, and three important pro inflammatory cytokines such as IL-6, IL-1 $\beta$ and TNF- $\alpha$. PRF's has ability to promote rapid angiogenesis and remodeling of wound tissue which is easier to adhere to connective tissue (Pochini, 2016).

However, in T2DM patients, the platelet quality is reported to be greatly decreased due to the state of chronic hyperglycemia. The additional HA in PRF might increase growth factor release in HAPRF compared with PRF alone. Ilio K, (2016) reported that HA can induce the release of growth factor from PRP in cases of genu osteoarthritisc

In HAPRF group, there is more increase of epithelialization tissue compared with PRF alone (Anderegg 2014). Therefore, the addition of HA, a matrix of extracellular components, known as anti-inflammatory, will control inflammation biomolecularly in the HAPRF combination (Azyenela R, 2015). HAPRF will make a moist environment in the DFU; it will support tissue regeneration. The combination of HA and GF contained in PRP has been published several times in various fields, both for the treatment of skin aging and cases of osteoarthritis. The combination of HA and PRP can reduce proinflammatory cytokines and increase the proliferation of articular chondrocytes and chondrogenic differentiation through the Erk1 / pathway. Meanwhile in PRP though Smad2 / 3 pathway. The clinical application of a mixture of PRP and HA may be more effective than PRP or HA alone for tissue regeneration ( Longitti, 2014).

HA scaffolding is used in tissue reconstruction techniques to provide a three-dimensional template for enhancing cell growth and GF supply. Delayed wound healing can be due to both reduced and excessive inflammation. Hyaluronic acid (hyaluronan, HA) is a large glycosaminoglycan and an essential extracellular component of skin. It is active throughout the entire process of wound healing being involved in proliferation, migration, and tissue remodeling. The combination of HA filler and Platelet concentrate can provide a synergistic effect, because HA acts as a scaffold and PRP induces collagen which is needed for wound repair (Chen WY,1999). There is a failure of chronic wound healing in T2DM patients and reduction of fibroblast function ( Azyenela Rika, 2016 ). Fibroblasts have decreased ability to proliferate and synthesize collagen and do not respond to the transforming growth factor1 (TGF-1). Platelet derived growth factor (PDGF) derived from platelet rich fibrin (PRF) lysates can restore TGF- $\beta$ receptor expression. Increasing the mechanical strength of the extracellular matrix with the addition of hyaluronic acid (HA) can improve TGF-beta signaling to trigger fibroblasts in wound epithelialization (Sundmann, 2014).

Longinotti, 2014 uses a combination of Platelet-Rich Plasma (PRP) and HA for treating open tendon wounds. In this study, HA synergized with PRP promoted rapid renovation and better healing, and a significant reduction in pain relief. Mateial HA has acts as an anti-inflammatory via tissue barrier by scavenging reactive oxygen species and inhibiting the inflammatory cell-derived serine proteinase. In addition, HA also has anti-edematous effect related to the osmotic HA buffer capacity( Chen et al, 1999).

Afat et al., (2017) reported that the combination of AH with L-PRF reduces edema after oral surgery for molar teeth 3. The HAPRF has a mechanism to reduce edema by means of HA affecting three main receptors in modulation of tissue regeneration, namely migration, proliferation and activation of keratinocyte cells (CD44). HAPRF also restores epidermal, fibroblast migration, controls inflammation, neoangiogenesis and promotes ECM deposits such as collagen fibers which contribute to wound healing (Price, 2005).

HA and PRF also work together to reduce inflammation due to chronic hyperglycaemia in diabetic patients (Sangameswaran, 2010).

That is to propose that mechanism of HAPRF in increased granulation tissue and epithelialization also reduces the inflammation. Due to decreased inflammation and wound repair, the combination HAPRF can indirectly reduce pain (Sreedam, 2012) (Figure 4).

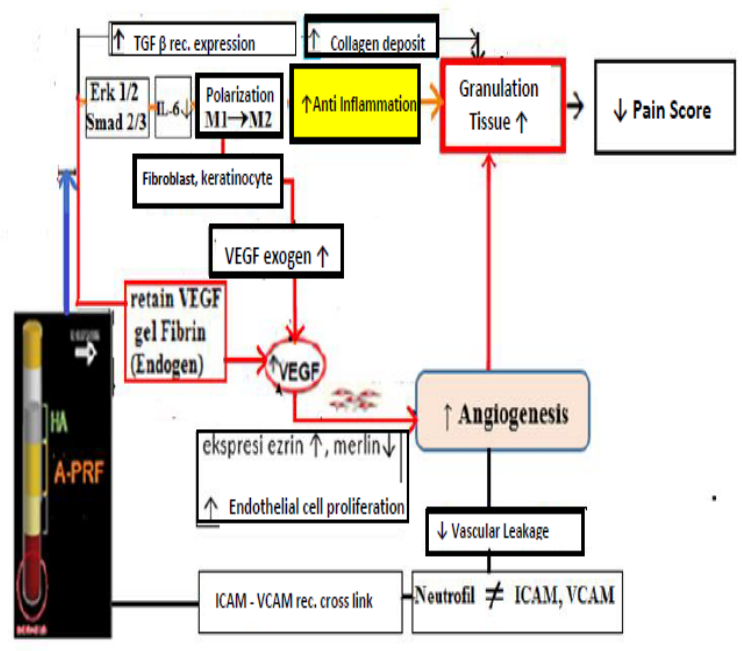

Figure 4. The proposed mechanism of HAPRF increases granulation tissue in DFU healing

\section{Conclusion}

Combination of HAPRF increase granulation tissue and epithelialization level on day-3 and day-7 significantly compared with PRF alone. It could also promote wound healing process in DFU by increasing angiogenesis, antiinflammatory and reduce pain. This would provide a new simple and cheap modality treatment for diabetic wounds in clinical practice.

\section{Acknowledge}

This study was a dissertation for a Program in Doctoral Medical Science Universitas Indonesia 


\section{Conflict of Interest}

This study was funded by Universitas Indonesia, Jakarta.

\section{References}

Armstrong DG et al .2017. Diabetic foot ulcers and their recurrence. N Engl J Med ,1(376):2367-75

Jeffcoate WJ et al.2018. Current challenges and opportunities in the prevention and management of diabetic foot ulcers. Diabetes Care , 41(1) :645-52

Abbott CA et al.2002. North-West Diabetes Foot Care Study. The North-West Diabetes Foot Care Study: incidence of, and risk factors for, new diabetic foot ulceration in a community-based patient cohort. Diabet Med ,19:377-84

Mills JL Sr et al. 2014.The Society for Vascular Surgery Lower Extremity Threatened Limb Classification System: risk stratification based on wound, ischemia, and foot infection (WIfI). J Vasc Surg , 59:220-34.e1-e2

Armstrong DG and Mills JL. 2013. Juggling risk to reduce amputations: the three-ring circus of infection, ischemia and tissue loss-dominant conditions. Wound Medicine ,1:13-14

Deng C et al.2017.Wound Repair Regen. Extracellular matrix/stromal vascular fraction gel conditioned medium accelerates wound healing in a murine model ,25(6):923-32. doi: 10.1111/wrr.12602.

Price RD.2005. The role of hyaluronic acid in wound healing: assessment of clinical evidence. Am J Clin Dermatol, 6(6):393-402.

Reksodiputro MH et al.2018. Platelet-rich fibrin enhances wound epithelialization in the skin graft donor site. J. Phys. Conf Ser.2(3):211-19

Pochini A et al.2016. Analysis of cytokine profile and growth factors in platelet-rich plasma obtained by open systems and commercial columns. Einstein (Sao Paulo), 14(3): 391-97.

Shi $\mathrm{G}$ et al.2018. Involvement of growth factors in diabetes mellitus and its complications: A general review, Biomed \& Pharmacother, 101(1): 510-27

Voigt J and Driver VR.2012.Hyaluronic acid derivatives and their healing effect on burns, epithelial surgical wounds, and chronic wounds: a systematic review and meta-analysis of randomized controlled trials. Wound Repair Regen,20(3):317-31.
Dohan Ehrenfest DM.2009. Slow release of growth factors and thrombospondins-1 in Choukroun's platelet-rich fibrin (PRF): a gold standard to achieve for all surgical platelet concentrates technologies. Growth factors , 27: 63-9

Ilio K.2016. Hyaloronic acid induces the release of growth factors from platelet rich plasma. Asia Pac J Sports Med Arthrosc Rehabil Technol, 4: 27-32.

Anderegg U et al.2014. More than just a filler - the role of hyaluronan for skin homeostasis. Experimental Dermatology, 23(5): 295-303

Azyenela R.2016. Efek Penambahan Asam Hialuronat Pada Lisat Platelet Rich Fibrin Terhadap Perbaikan Aktivitas Selular Fibroblas Tua, Gadjah Mada University Press,

Sundman EA et al. 2014.The anti-inflammatory and matrix restorative mechanisms of platelet-rich plasma in osteoarthritis. Am. J. Sports. Med,42(1), 35-41

Kartika RW, Alwi I, Suyatna FD, Yunir E, Waspadji S, Immanuel Suzzanna, et al. 2020.The Use of Image Processing in the Evaluation of Diabetic Foot Ulcer Granulation after Treatment with Advanced-Platelet Rich Fibrin + Hyaluronic Acid. Sys Rev Pharm., 11(12):519-526.

Longinotti C et al .2014. The use of hyaluronic acid based dressings to treat burns: A review. Burns Trauma , 2:162-74

Chen WY and Abatangelo G , 1999. Functions of hyaluronan in wound repair. Wound Repair Regen ,7:79-89

Afat IM et al .2018. Effects of leukocyte- and platelet-rich fibrin alone and combined with hyaluronic acid on pain, edema, and trismus after surgical extraction of impacted mandibular third molars. J Oral Maxillofac Surg,76(5):926-32

Sangameswaran B and Ilango K.2010. Evaluation Antihyperglycemic and antihyperlipidaemic activities of Andrographis leneata Nees on Stertpzotocin induced diabetic Rats, JJBS, 3(3):83-86

Sreedam Chandra Das et al .2012. Analgesic and Antiinflammatory Activities of Ethanolic Root Extract of Swertia chirata (Gentianaceae), 5 (1):31-36

Pastar I, Stojadinovic O, Yin NC, et al. Epithelialization in wound healing: a comprehensive review. Adv Wound Care (New Rochelle). 2014;3:445-464. 